\title{
The Association of Extreme Tachycardia and Sustained Return of Spontaneous Circulation after Nontraumatic Out-of-Hospital Cardiac Arrest
}

\author{
Dong Keon Lee $\mathbb{D}^{\mathbb{D}},{ }^{1}$ Eugi Jung, ${ }^{2}$ You Hwan Jo $\mathbb{D}^{1},{ }^{1,3}$ Joonghee Kim, ${ }^{1}$ Jae Hyuk Lee, \\ Seung Min Park $\mathbb{D}^{1}{ }^{1}$ and Yu Jin Kim ${ }^{1}$ \\ ${ }^{1}$ Department of Emergency Medicine, Seoul National University Bundang Hospital, Seongnam, Republic of Korea \\ ${ }^{2}$ Department of Emergency Medicine, VHS Medical Center, Seoul, Republic of Korea \\ ${ }^{3}$ Department of Emergency Medicine, Seoul National University College of Medicine, Seoul, Republic of Korea
}

Correspondence should be addressed to You Hwan Jo; emdrjyh@gmail.com

Received 15 March 2020; Revised 1 May 2020; Accepted 20 May 2020; Published 29 June 2020

Academic Editor: Chak W. Kam

Copyright (C) 2020 Dong Keon Lee et al. This is an open access article distributed under the Creative Commons Attribution License, which permits unrestricted use, distribution, and reproduction in any medium, provided the original work is properly cited.

\begin{abstract}
Objective. Heart rate (HR), an essential vital sign that reflects hemodynamic stability, is influenced by myocardial oxygen demand, coronary blood flow, and myocardial performance. HR at the time of the return of spontaneous circulation (ROSC) could be influenced by the $\beta 1$-adrenergic effect of the epinephrine administered during cardiopulmonary resuscitation (CPR), and its effect could be decreased in patients who have the failing heart. We aimed to investigate the association between $\mathrm{HR}$ at the time of ROSC and the outcomes of adult out-of-hospital cardiac arrest (OHCA) patients. Methods. This study was a secondary analysis of a cardiac arrest registry from a single institution from January 2008 to July 2014 . The OHCA patients who achieved ROSC at the emergency department (ED) were included, and HR was retrieved from an electrocardiogram or vital sign at the time of ROSC. The patients were categorized into four groups according to the $\mathrm{HR}$ (bradycardia $(\mathrm{HR}<60)$, normal $\mathrm{HR}(60 \leq \mathrm{HR} \leq 100)$, tachycardia $(100<\mathrm{HR}<150)$, and extreme tachycardia $(\mathrm{HR} \geq 150))$. The primary outcome was the rate of sustained ROSC and the secondary outcomes were the rate of one-month survival and six-month good neurologic outcome. Results. A total of 330 patients were included. In the univariate logistic regression model, the rate of sustained ROSC increased by $17 \%$ as HR increased by every 10 beats per minute (bpm) (odds ratio (OR), 1.171; 95\% confidence interval (CI), 1.077-1.274, $p<0.001$ ). In the multivariate logistic regression model, extreme tachycardia was independently associated with a high probability of sustained ROSC compared to normal heart rate (OR, 15.96; 95\% CI, 2.04-124.93, $p=0.008)$. Conclusion. Extreme tachycardia $(\mathrm{HR} \geq 150)$ at the time of ROSC is independently associated with a high probability of sustained ROSC in nontraumatic adult OHCA patients.
\end{abstract}

\section{Introduction}

Out-of-hospital cardiac arrest (OHCA) is still a major challenge in medicine despite many efforts to improve outcomes. In patients with OHCA, the rate of the return of spontaneous circulation (ROSC) has been reported to be from 24 to $44 \%$ [1-3]. Among patients who experience ROSC after OHCA, rearrest often develops within a short time and results in death.

Most in-hospital deaths after ROSC are due to cardiac dysfunction and neurologic failure in postcardiac arrest syndrome, and early death is mainly due to cardiac dysfunction [4-6]. Although cardiac dysfunction after ROSC is known to be reversible and cardiac function resumes after 48 to 72 hours, severe cardiac dysfunction prevents the maintenance of ROSC and may result in rearrest [7].

When ROSC is achieved, blood pressure and heart rate (HR) are usually measured immediately. HR, an essential vital sign that reflects hemodynamic stability, influences myocardial oxygen demand, coronary blood flow, and myocardial performance [8-10].

HR at the time of ROSC could be influenced by the $\beta 1$ adrenergic effect of the epinephrine administered during 
cardiopulmonary resuscitation (CPR), and its effect could be decreased in patients who have the failing heart which shows $\beta 1$-adrenergic receptor downregulation $[11,12]$.

In children with OHCA, it has been reported that $\mathrm{HR}$ in the first hour after sustained ROSC was associated with survival to discharge [13]. However, the association of $\mathrm{HR}$ at the time of ROSC with the outcomes of adult OHCA has yet to be investigated.

Given that the cardiac dysfunction after ROSC showed significant morbidity and mortality [14], we hypothesized that the HR at the time of ROSC, which might reflect the cardiac function, could be associated with the outcome of OHCA. Therefore, we investigated the association between $\mathrm{HR}$ at the time of ROSC and the probability of sustained ROSC.

\section{Methods}

2.1. Study Design and Setting. We performed a secondary analysis of consecutive OHCA databases in a single ED from January 2008 to July 2014. The study facility was a 1250-bed urban tertiary academic hospital with an annual ED census of approximately 85,000 . This study was approved by the Institutional Review Board (IRB) of Seoul National University Bundang Hospital and reported according to the STROBE (STrengthening the Reporting of OBservational studies in Epidemiology) guidelines for reporting observational trials [15].

Patients with cardiac arrest were managed based on the recommendations of the international guidelines during the study period [16]. Prehospital CPR was provided by firestation-based emergency medical technicians (EMTs), and the level of prehospital CPR was primarily basic life support, including chest compression/ventilation and automatic external defibrillator use.

When the prehospital EMTs notified the ED, the CPR team of the ED was activated. Details on the CPR team and resuscitation protocol in the $\mathrm{ED}$ have been presented previously [17]. The standard-dose epinephrine defined as $1 \mathrm{mg}$ was administered intravenously every three to five minutes [16]. Blood samples were drawn during the CPR and immediately after ROSC, and they were then sent to the central laboratory for analysis. When ROSC was achieved, the vital signs were measured with noninvasive equipment (IntelliVue, MX700, Philips, Netherlands) within three minutes after ROSC and every one hour thereafter. Among them, the first measurement was used for analysis. The 12-lead electrocardiography (ECG) (Philips PageWriter TC-70; GE, Eindhoven, the Netherlands) was also taken within three minutes after ROSC. If there was a clinical necessity, vital signs were measured and ECG was taken repeatedly.

2.2. Study Population. Based on the OHCA registry in accordance with Utstein-style guidelines [18], consecutive patients who were 18 years or older with nontraumatic OHCA and received advanced cardiac life support in the ED were included in this study $[19,20]$. Patients under 18 years, patients who achieved prehospital ROSC or failed to achieve any ROSC, patients with traumatic cardiac arrest, patients without recorded $\mathrm{HR}$ due to brief ROSC of less than a minute, and patients who were rescued by extracorporeal life support (ECLS) were excluded.

Bradycardia is defined as HR below 60 beats per minute and tachycardia is defined as HR above 100 beats per minute. Given those definitions and that symptomatic tachycardia generally involves rates over 150 beats/min [21-23], the patients were categorized into four groups according to their $\mathrm{HR}$ at the time of ROSC as follows: bradycardia $(\mathrm{HR}<60$ beats/min), normal HR $(60 \leq \mathrm{HR} \leq 100$ beats/min), tachycardia $(100<\mathrm{HR}<150$ beats/min), and extreme tachycardia (HR $\geq 150$ beats/min).

2.3. Data Collection and Outcomes. Data were collected by trained research personnel using a standardized registry template form [18]. The form included demographics, witnessed arrest, place of arrest, bystander CPR, initial rhythm, presumed cause of arrest, drugs, and the outcomes of the patient including sustained ROSC, one-month survival, and six-month neurologic outcome.

The HR of each patient was retrieved from the ECG taken at the time of ROSC. If the HR was not available from the ECG, the HR was retrieved from the vital signs recorded immediately after ROSC. $N$-terminal pro-brain type natriuretic peptide (NT-proBNP) was measured with blood samples taken immediately after ROSC in the central laboratory to investigate the association between the NTproBNP concentration and the HR groups at the time of ROSC.

The primary outcome was the rate of sustained ROSC according to the HR groups. Sustained ROSC was defined according to the Utstein statement as the status in which chest compressions for 20 consecutive minutes are not required and signs of circulation persist [24]. The secondary outcomes were one-month survival and six-month good neurologic outcome according to the HR groups. The sixmonth good neurologic outcome was defined as a cerebral performance category (CPC) one or two at six months after admission, and it was investigated with structured telephone follow-up protocol performed by trained research personnel.

2.4. Statistical Analyses. The Chi-square test or Fisher's exact test was used for comparisons of categorical variables and presented as numbers (percentages). Continuous variables were examined with the Kolmogorov-Smirnov test for the normality of the distribution and presented as medians (interquartile ranges). The analysis of variance or the Kruskal-Wallis test was used depending on the normality of the distribution. If the Kruskal-Wallis test showed statistical significance, Bonferroni correction was used for multiple comparisons.

Univariable logistic regression analysis was performed to investigate the association between the HR (as a continuous variable) at the time of ROSC and the probability of sustained ROSC. Multivariable logistic regression analysis was performed with the enter method, which brings all clinically relevant variables into the model, to determine the 


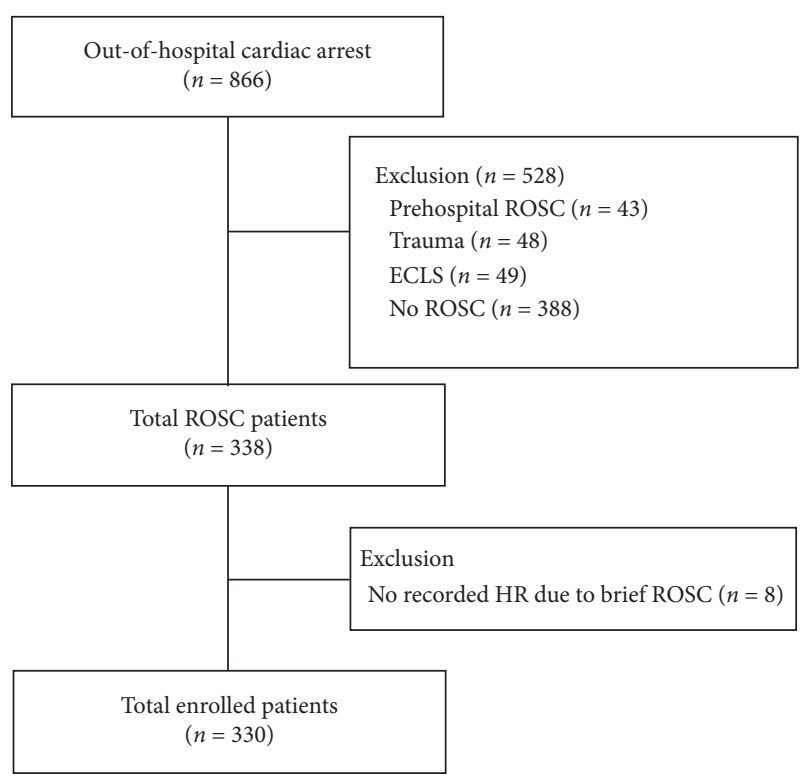

FIGURE 1: Flowchart of the study population. ROSC, return of spontaneous circulation; ECLS, extracorporeal life support; HR, heart rate; brief ROSC, ROSC less than one minute of duration.

independent factors for outcomes including sustained ROSC, one-month survival, and six-month neurologic outcome. As a result, HR (bradycardia, normal HR, tachycardia, and extreme tachycardia), age, sex, witnessed arrest, public place, shockable initial rhythm, bystander $\mathrm{CPR}$, total epinephrine dose, mean arterial pressure at the time of ROSC, no-flow time defined as time from collapse to the start of resuscitation attempts, and low-flow time defined as time from start of life support until the restoration of spontaneous circulation were included in the multivariable logistic regression analysis.

All tests were two-sided, and $p$ values $<0.05$ were considered to indicate statistical significance. Data were analyzed using IBM SPSS statistics version 21 (IBM, Armonk, NY, USA), and a curve fit was performed with ggplot2 software.

\section{Results}

3.1. Baseline Characteristics. During the study period, a total of 866 adult OHCA patients who presented to the ED were initially screened. Among these patients, 43 patients who achieved prehospital ROSC, 48 patients whose cause of cardiac arrest was trauma, 49 patients who were supported by ECLS devices, and 388 patients who did not achieve any ROSC were excluded. Among the remaining 338 patients, eight had no HR record because of brief ROSC (duration less than one minute). As a result, 330 OHCA patients who underwent CPR in the ED and had HR records after ROSC were included in the final analysis (Figure 1).

The median age of the patients was 72 (59-79) years, and $194(58.8 \%)$ patients were males. HR was retrieved from the ECGs taken immediately after ROSC in 323 patients and from the vital signs in seven patients. The numbers of patients according to HR were 26 in the bradycardia group, 67 in the normal HR group, 176 in the tachycardia group, and 61 in the extreme tachycardia group. The mean arterial pressure at the time of ROSC was higher in the extreme tachycardia group than in the other groups. The total epinephrine dose was lower in the extreme tachycardia group compared to the other groups. The median low-flow time showed no significant difference between groups after Bonferroni correction. There was no significant difference among groups in terms of the age, gender, rates of witnessed arrest, public place, initial shockable rhythm, bystander $\mathrm{CPR}$, and no-flow time (Table 1).

\subsection{Resuscitation Outcomes according to the HR Groups.} In terms of the outcomes, the sustained ROSC was achieved in 60 of 61 patients $(98.4 \%)$ in the extreme tachycardia group, and the rate of the sustained ROSC was highest in the extreme tachycardia group and lowest in the bradycardia group (Table 1). In the univariable logistic regression model, the probability of sustained ROSC increased by $17 \%$ as HR increased by every 10 beats per minute (OR, 1.171, 95\% confidence interval [CI], 1.077-1.274, $p<0.001$ ) (Figure 2). The rates of one-month survival and six-month good neurologic outcome were not different between the four groups (Table 1).

In the multivariable logistic regression analysis, the extreme tachycardia group was independently associated with a high probability of sustained ROSC compared to the normal HR group (OR 11.297; 95\% CI 1.350-94.527; $p=0.025$, Table 2).

3.3. NT-proBNP Concentration and the HR Groups. The median NT-proBNP concentration in total patients was 1403 [277-4579] pg/mL. The NT-proBNP concentration was significantly lower in the extreme tachycardia group than in the normal HR group (874 [164-2902] pg/mL and 2277 [519-8743] $\mathrm{pg} / \mathrm{ml}$ in the extreme tachycardia group and normal HR group, respectively; $p=0.043$; Figure 3). There was no difference between the bradycardia, normal $\mathrm{HR}$, and tachycardia groups.

\section{Discussion}

In the present study, patients with extreme tachycardia $(\mathrm{HR} \geq 150$ beats/min) had the highest rate of sustained ROSC, and extreme tachycardia was an independent factor for the probability of sustained ROSC.

The myocardium is damaged by ischemia and reperfusion injury and electrical shock during cardiac arrest and resuscitation. It leads to reduced contractility and compliance of myocardium, and severe myocardial dysfunction has been reported to be associated with high mortality in the early postcardiac arrest period $[25,26]$.

Myocardial dysfunction after cardiac arrest has been measured directly or indirectly using pulmonary artery catheterization, echocardiography, blood pressure, and several biomarkers such as troponin and NT-proBNP concentrations. Low cardiac output measured by pulmonary artery catheterization was common in patients after ROSC 
TABLE 1: Baseline characteristics and the outcomes of the patients.

\begin{tabular}{|c|c|c|c|c|c|c|}
\hline & $\begin{array}{c}\text { Total } \\
(n=330)\end{array}$ & $\begin{array}{c}\text { Bradycardia } \\
\quad(n=26)\end{array}$ & $\begin{array}{l}\text { Normal HR } \\
\quad(n=67)\end{array}$ & $\begin{array}{c}\text { Tachycardia } \\
(n=176)\end{array}$ & $\begin{array}{l}\text { Extreme tachycardia } \\
\qquad(n=61)\end{array}$ & $p$ \\
\hline Age (years) & $72(59-79)$ & $73(66-77)$ & $74(62-79)$ & $72(60-79)$ & $69(49-76)$ & 0.094 \\
\hline Male & $194(58.8)$ & $17(65.4)$ & $44(65.7)$ & $98(55.7)$ & $35(57.4)$ & 0.470 \\
\hline Witnessed arrest & $264(80.0)$ & $20(76.9)$ & $57(85.1)$ & $141(80.1)$ & $46(75.4)$ & 0.565 \\
\hline Public place & $52(15.8)$ & $5(19.2)$ & $11(16.4)$ & $23(13.1)$ & $13(21.3)$ & 0.451 \\
\hline Shockable initial rhythm & $41(12.4)$ & $3(11.5)$ & $7(10.4)$ & $21(11.9)$ & $10(16.4)$ & 0.757 \\
\hline Bystander CPR & $105(31.8)$ & $10(38.5)$ & $18(26.9)$ & $55(31.3)$ & $22(36.1)$ & 0.611 \\
\hline MAP at ROSC $(\mathrm{mmHg})$ & $93(68-114)$ & $81(43-94)$ & $89(67-105)$ & $89(68-113)$ & $111(85-137)$ & $<0.001^{*}$ \\
\hline $\mathrm{HR}$ at ROSC (beats/min) & $120(98-144)$ & $47(32-51)$ & $84(76-94)$ & $124(113-136)$ & $161(155-175)$ & $<0.001^{*}$ \\
\hline No-flow time (min) & $4(0-12)$ & $4(1-14)$ & $5(0-12)$ & $4(0-11)$ & $1(0-9)$ & 0.194 \\
\hline Low-flow time (min) & $26(17-36)$ & $37(18-46)$ & $27(18-36)$ & $25(17-34)$ & $24(15-32)$ & $0.047^{\dagger}$ \\
\hline Total epinephrine dose (mg) & $3(2-4)$ & $3(2-7)$ & $4(2-5)$ & $3(2-4)$ & $2(1-3)$ & $<0.001^{*}$ \\
\hline Sustained ROSC & $281(85.2)$ & $18(69.2)$ & $54(80.6)$ & $149(84.7)$ & $60(98.4)$ & $0.002^{\ddagger}$ \\
\hline One-month survival & $58(17.8)$ & $4(15.4)$ & $11(16.1)$ & $26(15.1)$ & $17(27.9)$ & 0.153 \\
\hline Good neurologic outcome & $23(7.0)$ & $2(7.7)$ & $4(6.0)$ & $5(6.0)$ & $5(6.0)$ & 0.965 \\
\hline
\end{tabular}

Data are expressed as median (interquartile range) or number (\%) as appropriate. Bradycardia, $\mathrm{HR}<60$ beats/min; normal, $60 \leq \mathrm{HR} \leq 100 \mathrm{beats} / \mathrm{min}$; tachycardia, $100<\mathrm{HR}<150$ beats/min; extreme tachycardia, HR $\geq 150$ beats/min; HR, heart rate; CPR, cardiopulmonary resuscitation; MAP, mean arterial pressure; ROSC, return of spontaneous circulation. ${ }^{*}$ Statistically significant after Bonferroni correction. ${ }^{\dagger}$ Statistically not significant after Bonferroni correction. ${ }^{\ddagger}$ Statistically significant.

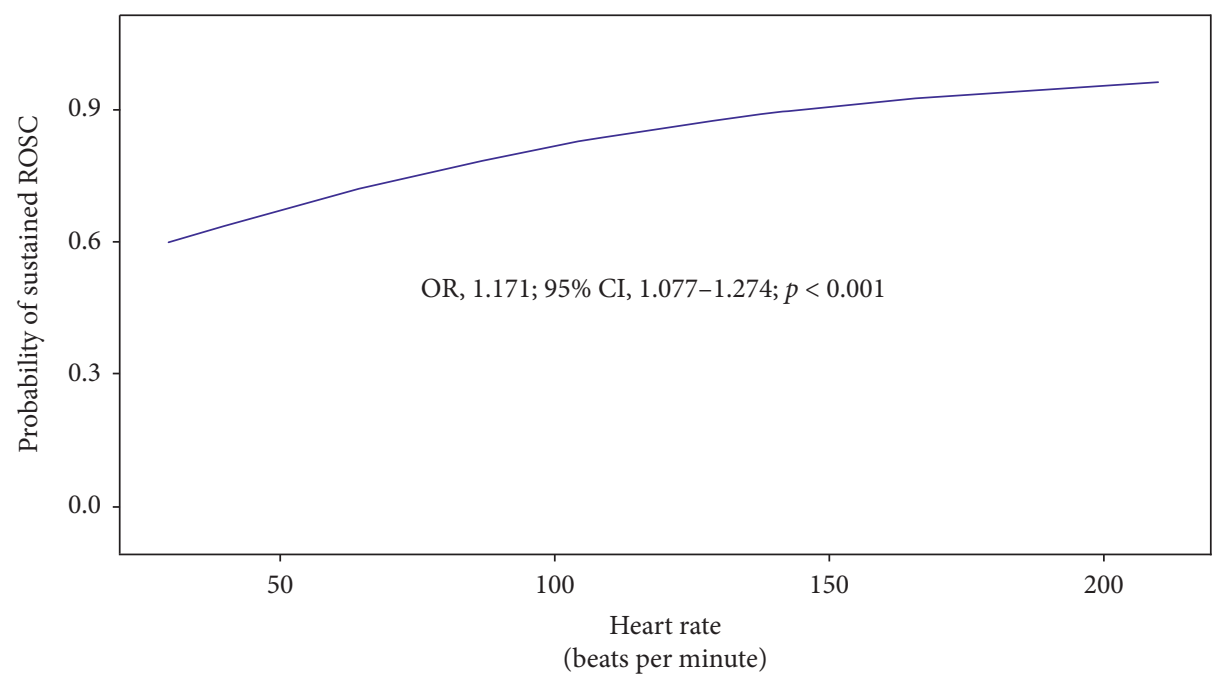

FIGURE 2: The probability of sustained ROSC according to heart rate. ROSC, return of spontaneous circulation.

from cardiac arrest, but the association of cardiac output with outcomes was unclear $[27,28]$. Echocardiography has been widely used to measure the myocardial function after ROSC. The systolic and diastolic dysfunctions were observed after ROSC, and they were associated with outcomes [7, 26, 29]. Hypotension and vasopressor support after ROSC were associated with ischemic insult leading to cardiovascular failure, which could predict worse outcomes after cardiac arrest [30-32]. Troponin and NT-proBNP have also been investigated in patients with cardiac arrest although the results were reported differently at the time of measurement [33].

In this study, extreme tachycardia was associated with the high probability of sustained ROSC. However, Redwood et al. reported that higher HR in acute myocardial ischemia was associated with increased myocardial infarct size in the experimental model, while reduced HR may show a beneficial effect because of the decrease in myocardial oxygen consumption and prolongation of total diastolic time which affects coronary collateral flow [34].

This difference could be explained by the $\beta 1$-adrenergic receptor downregulation. Epinephrine is an $\alpha$ - and $\beta$-adrenoceptor agonist and was administered every three minutes during CPR [35]. The $\alpha$-adrenergic effect increases diastolic aortic pressure and coronary and cerebral perfusion pressure, whereas the $\beta 1$-adrenergic effect increases HR and contractility $[21,29]$. Given that $\beta 1$-adrenergic receptor downregulation occurs in postcardiac arrest myocardial dysfunction and failing heart $[11,12]$, extreme tachycardia could be the response of epinephrine in the better-conditioned heart. This is supported by the lowest NT-proBNP concentration of the extreme tachycardia group. Hence, the patients who showed extreme tachycardia at the time of ROSC might have better-conditioned hearts that show preserved $\beta 1$-adrenergic receptors and concomitant better responses to epinephrine than the others. 
TABLe 2: Multivariable logistic regression analysis for the outcomes.

\begin{tabular}{|c|c|c|c|c|c|c|c|c|c|}
\hline & \multicolumn{3}{|c|}{ Sustained ROSC } & \multicolumn{3}{|c|}{ One-month survival } & \multicolumn{3}{|c|}{$\begin{array}{l}\text { Six-month good neurologic } \\
\text { outcome }\end{array}$} \\
\hline & OR & $(95 \% \mathrm{CI})$ & $p$ & OR & $(95 \% \mathrm{CI})$ & $p$ & OR & (95\% CI) & $p$ \\
\hline \multicolumn{10}{|l|}{ Heart rate group } \\
\hline Bradycardia & 0.79 & $(0.23-2.75)$ & 0.711 & 1.92 & $(0.41-9.10)$ & 0.411 & 1.69 & $(0.29-9.82)$ & 0.558 \\
\hline Normal HR & 1.00 & (Reference) & & 1.00 & (Reference) & & 1.00 & (Reference) & \\
\hline Tachycardia & 1.23 & $(0.54-2.82)$ & 0.624 & 0.70 & $(0.27-1.79)$ & 0.449 & 0.79 & $(0.29-2.16)$ & 0.642 \\
\hline Extreme tachycardia & 11.30 & $(1.35-94.53)$ & $0.025^{*}$ & 0.82 & $(0.27-2.49)$ & 0.724 & 0.92 & $(0.28-3.08)$ & 0.896 \\
\hline Age, per 10 years & 0.85 & $(0.66-1.11)$ & 0.230 & 0.82 & $(0.66-1.02)$ & 0.079 & 0.80 & $(0.63-1.02)$ & 0.073 \\
\hline Male & 0.73 & $(0.34-1.57)$ & 0.427 & 2.15 & $(1.02-4.55)$ & $0.045^{*}$ & 3.45 & $(1.56-21.50)$ & $0.009^{*}$ \\
\hline Witnessed arrest & 2.15 & $(0.91-5.11)$ & 0.083 & 1.30 & $(0.49-3.46)$ & 0.605 & 0.96 & $(0.34-2.65)$ & 0.929 \\
\hline Public place & 1.84 & $(0.56-6.02)$ & 0.313 & 1.11 & $(0.42-2.96)$ & 0.829 & 1.83 & $(0.66-5.07)$ & 0.245 \\
\hline Shockable initial rhythm & 1.19 & $(0.37-3.84)$ & 0.268 & 3.03 & $(1.22-7.51)$ & $0.017^{*}$ & 3.18 & $(1.25-8.10)$ & $0.015^{*}$ \\
\hline Bystander CPR & 1.67 & $(0.67-4.16)$ & 0.268 & 1.81 & $(0.76-4.34)$ & 0.182 & 1.87 & $(0.71-4.91)$ & 0.205 \\
\hline Total epinephrine dose, per $1 \mathrm{mg}$ & 0.89 & $(0.75-1.05)$ & 0.152 & 0.69 & $(0.52-0.92)$ & 0.011 & 0.77 & $(0.57-1.04)$ & 0.083 \\
\hline $\mathrm{MAP} \geq 80 \mathrm{mmHg}$ at ROSC & 0.90 & $(0.43-1.88)$ & 0.778 & 2.21 & $(0.98-4.97)$ & 0.056 & 1.84 & $(0.76-4.43)$ & 0.175 \\
\hline No-flow time, per $1 \mathrm{~min}$ & 1.02 & $(0.98-1.06)$ & 0.395 & 0.95 & $(0.91-1.00)$ & 0.057 & 0.94 & $(0.89-0.99)$ & $0.042^{*}$ \\
\hline Low-flow time, per $1 \mathrm{~min}$ & 0.99 & $(0.97-1.02)$ & 0.716 & 0.95 & $(0.91-0.98)$ & $0.005^{*}$ & 0.94 & $(0.91-0.98)$ & $0.007^{*}$ \\
\hline
\end{tabular}

Bradycardia, HR $<60$ beats/min; normal, $60 \leq \mathrm{HR} \leq 100$ beats/min; tachycardia, $100<\mathrm{HR}<150$ beats/min; extreme tachycardia, HR $\geq 150$ beats/min. ROSC, return of spontaneous circulation; $\mathrm{OR}$, odds ratio; $\mathrm{CI}$, confidence interval; HR, heart rate; CPR, cardiopulmonary resuscitation; MAP, mean arterial pressure. ${ }^{*}$ Statistically significant.

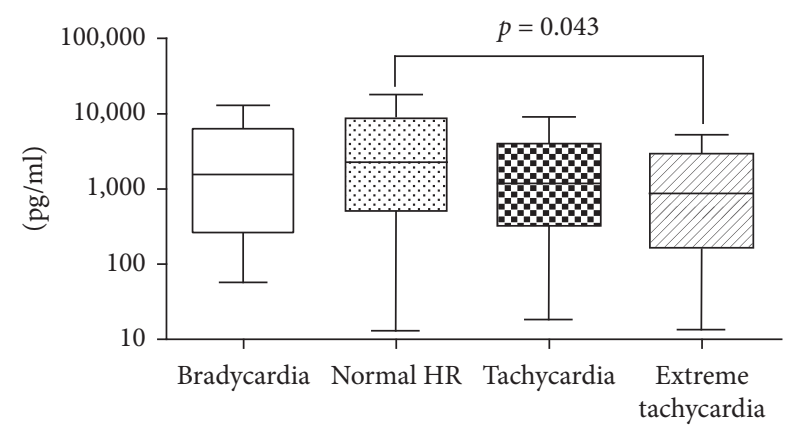

FIGURE 3: $N$-terminal pro-brain-type natriuretic peptide concentration. Bradycardia, heart rate $(\mathrm{HR})<60$ beats/min; normal, $60 \leq \mathrm{HR} \leq 100$ beats/min; tachycardia, $100<\mathrm{HR}<150$ beats/min; extreme tachycardia, HR $\geq 150$ beats $/ \mathrm{min}$.

Although extreme tachycardia showed a high rate of sustained ROSC, it showed no association with the sixmonth good neurologic outcome. Nevertheless, HR is still important since early prognostication is valuable to the determination of the treatment and disposition plan for OHCA patients, and it is an easily and immediately measurable parameter. Based on the results of the present study, patients with extreme tachycardia after ROSC should be paid more attention in regard to postresuscitation care such as primary coronary intervention and targeted temperature management, while hemodynamic support should be continued for all patients including those without extreme tachycardia after ROSC.

This study has some limitations. First, this study was performed retrospectively in a single institution. Therefore, the results might not be generalizable to other institutions, so a multicenter study is warranted.

Second, echocardiography was not performed immediately after ROSC, so the exact cardiac function could not be investigated. Instead, NT-proBNP was used as an indicator of cardiac function.

Third, we analyzed HR only at the time of ROSC and did not analyze the change with time. Depending on cardiac function and type and dose of vasopressors and inotropes, HR could be different with time. However, although we mainly retrieved HR from the ECG taken immediately after ROSC, HR could be easily measured with cardiac monitoring. Therefore, it is worth noting that a simple HR measurement could be used as an independent predictor of sustained ROSC.

\section{Conclusions}

Extreme tachycardia $(\mathrm{HR} \geq 150)$ at the time of ROSC is associated with a high probability of sustained ROSC in nontraumatic adult OHCA patients.

\section{Data Availability}

The data used to support the findings of this study have not been made available because the authors were not allowed to share the patients' information.

\section{Conflicts of Interest}

The authors declare that they have no conflicts of interest regarding the publication of this article.

\section{References}

[1] J.-T. Gräsner, P. Meybohm, R. Lefering et al., "ROSC after cardiac arrest-the RACA score to predict outcome after outof-hospital cardiac arrest," European Heart Journal, vol. 32, no. 13, pp. 1649-1656, 2011.

[2] Y. S. Ro, S. D. Shin, K. J. Song et al., "A trend in epidemiology and outcomes of out-of-hospital cardiac arrest by urbanization level: a nationwide observational study from 2006 to 2010 
in South Korea," Resuscitation, vol. 84, no. 5, pp. 547-557, 2013.

[3] D. W. Spaite, B. J. Bobrow, T. F. Vadeboncoeur et al., "The impact of prehospital transport interval on survival in out-ofhospital cardiac arrest: implications for regionalization of post-resuscitation care," Resuscitation, vol. 79, no. 1, pp. 61-66, 2008.

[4] S. J. Lewis, "Survival after failed out-of-hospital resuscitation," The Journal of Emergency Medicine, vol. 13, no. 3, p. 427, 1995.

[5] S. Laver, C. Farrow, D. Turner, and J. Nolan, "Mode of death after admission to an intensive care unit following cardiac arrest," Intensive Care Medicine, vol. 30, no. 11, pp. 21262128, 2004.

[6] W.-T. Chang, M. H.-M. Ma, K.-L. Chien et al., "Postresuscitation myocardial dysfunction: correlated factors and prognostic implications," Intensive Care Medicine, vol. 33, no. 1, pp. 88-95, 2007.

[7] K. B. Kern, R. W. Hilwig, R. A. Berg et al., "Postresuscitation left ventricular systolic and diastolic dysfunction," Circulation, vol. 95, no. 12, pp. 2610-2613, 1997.

[8] G. Heusch, "Heart rate in the pathophysiology of coronary blood flow and myocardial ischaemia: benefit from selective bradycardic agents," British Journal of Pharmacology, vol. 153, no. 8, pp. 1589-1601, 2008.

[9] J.-C. Reil, F. Custodis, K. Swedberg et al., "Heart rate reduction in cardiovascular disease and therapy," Clinical Research in Cardiology, vol. 100, no. 1, pp. 11-19, 2011.

[10] J.-C. Reil and M. Böhm, "The role of heart rate in the development of cardiovascular disease," Clinical Research in Cardiology, vol. 96, no. 9, pp. 585-592, 2007.

[11] M. R. Bristow, "Changes in myocardial and vascular receptors in heart failure," Journal of the American College of Cardiology, vol. 22, no. 4, pp. A61-A71, 1993.

[12] X.-F. Ji, S. Shuo Wang, L. Yang, and C.-S. Li, "Impaired $\beta$-adrenergic receptor signalling in post-resuscitation myocardial dysfunction," Resuscitation, vol. 83, no. 5, pp. 640-644, 2012.

[13] Y.-R. Lin, C.-J. Li, T.-K. Wu et al., "Post-resuscitative clinical features in the first hour after achieving sustained ROSC predict the duration of survival in children with non-traumatic out-of-hospital cardiac arrest," Resuscitation, vol. 81, no. 4, pp. 410-417, 2010.

[14] D. Stub, S. Bernard, S. J. Duffy, and D. M. Kaye, "Post cardiac arrest syndrome," Circulation, vol. 123, no. 13, pp. 1428-1435, 2011.

[15] E. von Elm, D. G. Altman, M. Egger et al., “The strengthening the reporting of observational studies in epidemiology (STROBE) statement: guidelines for reporting observational studies," Annals of Internal Medicine, vol. 147, no. 8, pp. 573-577, 2007.

[16] R. W. Neumar, C. W. Otto, M. S. Link et al., "Part 8: adult advanced cardiovascular life support: 2010 American heart association guidelines for cardiopulmonary resuscitation and emergency cardiovascular care," Circulation, vol. 122, no. 18 suppl_3, pp. S729-S767, 2010.

[17] J. Kang, J. Kim, Y. H. Jo et al., "ED crowding and the outcomes of out-of-hospital cardiac arrest," The American Journal of Emergency Medicine, vol. 33, no. 11, pp. 1659-1664, 2015.

[18] G. D. Perkins, I. G. Jacobs, V. M. Nadkarni et al., "Cardiac arrest and cardiopulmonary resuscitation outcome reports: update of the Utstein resuscitation registry templates for outof-hospital cardiac arrest: a statement for healthcare professionals from a task force of the international liaison committee on resuscitation (American heart association,
European resuscitation council, Australian and New Zealand council on resuscitation, heart and stroke foundation of Canada, Inter American Heart Foundation, resuscitation council of southern africa, resuscitation council of asia); and the American heart association emergency cardiovascular care committee and the council on cardiopulmonary, critical care, perioperative and resuscitation," Resuscitation, vol. 132, no. 13 , pp. 1286-3000, 2015.

[19] J. Kim, K. Kim, T. Kim et al., "The clinical significance of a failed initial intubation attempt during emergency department resuscitation of out-of-hospital cardiac arrest patients," Resuscitation, vol. 85, no. 5, pp. 623-627, 2014.

[20] J. Kim, K. Kim, C. W. Callaway et al., "Dynamic prediction of patient outcomes during ongoing cardiopulmonary resuscitation," Resuscitation, vol. 111, pp. 127-133, 2017.

[21] M. S. Link, L. C. Berkow, P. J. Kudenchuk et al., "Part 7: adult advanced cardiovascular life support," Circulation, vol. 132, no. 18 suppl 2, pp. S444-S464, 2015.

[22] J. Soar, J. P. Nolan, B. W. Böttiger et al., "European resuscitation council guidelines for resuscitation 2015," Resuscitation, vol. 95, pp. 100-147, 2015.

[23] F. M. Kusumoto, M. H. Schoenfeld, C. Barrett et al., "2018 ACC/AHA/HRS guideline on the evaluation and management of patients with bradycardia and cardiac conduction delay," Journal of the American College of Cardiology, vol. 74, no. 7, 2018.

[24] I. Jacobs, V. Nadkarni, J. Bahr et al., "Cardiac arrest and cardiopulmonary resuscitation outcome reports," Circulation, vol. 110, no. 21, pp. 3385-3397, 2004.

[25] R. W. Neumar, J. P. Nolan, C. Adrie et al., "Post--cardiac arrest syndrome," Circulation, vol. 118, no. 23, pp. 2452-2483, 2008.

[26] K. B. Kern, R. W. Hilwig, K. H. Rhee, and R. A. Berg, "Myocardial dysfunction after resuscitation from cardiac arrest: an example of global myocardial stunning," Journal of the American College of Cardiology, vol. 28, no. 1, pp. 232-240, 1996.

[27] I. Laurent, M. Monchi, J.-D. Chiche et al., "Reversible myocardial dysfunction in survivors of out-of-hospital cardiac arrest," Journal of the American College of Cardiology, vol. 40, no. 12, pp. 2110-2116, 2002.

[28] S. Trzeciak, A. E. Jones, J. H. Kilgannon et al., "Significance of arterial hypotension after resuscitation from cardiac arrest," Critical Care Medicine, vol. 37, no. 11, pp. 2895-2903, 2009.

[29] E. R. Gonzalez, B. S. Kannewurf, and M. L. Hess, Inotropic Therapy and the Critically Ill Patient. Textbook of Critical Care, pp. 1123-1130, WB Saunders, Philadelphia, PA, USA, 2000.

[30] J. Bro-Jeppesen, M. Annborn, C. Hassager et al., "Hemodynamics and vasopressor support during targeted temperature management at $33^{\circ} \mathrm{C}$ versus $36^{\circ} \mathrm{C}$ after out-of-hospital cardiac arrest," Critical Care Medicine, vol. 43, no. 2, pp. 318-327, 2015.

[31] K. Fox, I. Ford, P. G. Steg, M. Tendera, M. Robertson, and R. Ferrari, "Heart rate as a prognostic risk factor in patients with coronary artery disease and left-ventricular systolic dysfunction (BEAUTIFUL): a subgroup analysis of a randomised controlled trial," The Lancet, vol. 372, no. 9641, pp. 817-821, 2008.

[32] M. W. Donnino, L. W. Andersen, T. Giberson et al., "Initial lactate and lactate change in post-cardiac arrest," Critical Care Medicine, vol. 42, no. 8, pp. 1804-1811, 2014.

[33] P. L. Myhre, M. Tiainen, V. Pettilä et al., "NT-proBNP in patients with out-of-hospital cardiac arrest: results from the 
FINNRESUSCI Study,” Resuscitation, vol. 104, pp. 12-18, 2016.

[34] D. R. Redwood, E. R. Smith, and S. E. Epstein, "Coronary artery occlusion in the conscious dog," Circulation, vol. 46, no. 2, pp. 323-332, 1972.

[35] V. R. Kee, "Hemodynamic pharmacology of intravenous vasopressors," Critical Care Nurse, vol. 23, no. 4, pp. 79-82, 2003. 